\title{
Robust scalable reversible strong adhesion by gecko-inspired composite design
}

\author{
Xiaosong LI, Pengpeng BAI, Xinxin LI, Lvzhou LI, Yuanzhe LI, Hongyu LU, Liran MA*, Yonggang MENG, \\ Yu TIAN* \\ State Key Laboratory of Tribology, Department of Mechanical Engineering, Tsinghua University, Beijing 100084, China \\ Received: 14 October 2020 / Revised: 13 January 2021 / Accepted: 19 April 2021 \\ (C) The author(s) 2021.
}

\begin{abstract}
Bio-inspired reversible adhesion has significant potential in many fields requiring flexible grasping and manipulation, such as precision manufacturing, flexible electronics, and intelligent robotics. Despite extensive efforts for adhesive synthesis with a high adhesion strength at the interface, an effective strategy to actively tune the adhesion capacity between a strong attachment and an easy detachment spanning a wide range of scales has been lagged. Herein, we report a novel soft-hard-soft sandwiched composite design to achieve a stable, repeatable, and reversible strong adhesion with an easily scalable performance for a large area ranging from $\sim 1.5$ to $150 \mathrm{~cm}^{2}$ and a high load ranging from $\sim 20$ to $700 \mathrm{~N}$. Theoretical studies indicate that this design can enhance the uniform loading for attachment by restraining the lateral shrinkage in the natural state, while facilitate a flexible peeling for detachment by causing stress concentration in the bending state, yielding an adhesion switching ratio of $\sim 54$ and a switching time of less than $\sim 0.2 \mathrm{~s}$. This design is further integrated into versatile grippers, climbing robots, and human climbing grippers, demonstrating its robust scalability for a reversible strong adhesion. This biomimetic design bridges microscopic interfacial interactions with macroscopic controllable applications, providing a universal and feasible paradigm for adhesion design and control.
\end{abstract}

Keywords: biomimetic design; reversible adhesion; composite; cross-scale; robotics

\section{Introduction}

Adhesion is a ubiquitous interfacial phenomenon found in nature (e.g., in geckos and spiders) and daily life (e.g., adhesives and glue), spanning a wide spatial scale of several orders of magnitude, such as nanoscopic gecko spatula, microscopic cell adhesion, and macroscopic building coating [1-6]. It is mainly determined by intermolecular interactions at the interface, which are affected by the intrinsic material properties (e.g., surface energy and Young's modulus) and geometric structural properties (e.g., layer thickness and shape of the structure) [7-10]. Many biological samples, such as geckos, beetles, and mussels, have evolved unique organs and elaborate structures to achieve attachment to various substrates. As a paragon of adhesion control, geckos can firmly adhere to various surfaces through van der Waals interactions originating from the hierarchical structures on their toes, while quickly release their toes through a peeling motion [11-14]. Gecko adhesion is actively reversible through muscle modulation, is effective for most surfaces without leaving any residuals, and achieves a long durability, thereby exhibiting an ideal performance for applications requiring flexible grasping and manipulation. Inspired by the remarkable properties of gecko adhesion, extensive efforts have been made to develop technologies for reversible adhesion, propelling advances in related technologies such as precision manufacturing, flexible electronics, robotics, and aeronautics [15-21].

Focusing on the sophisticated micro-/nano-hairs on

* Corresponding authors: Yu TIAN, E-mail: tianyu@mail.tsinghua.edu.cn; Liran MA, E-mail: maliran@mail.tsinghua.edu.cn 
gecko toes, tremendous bio-inspired adhesive surfaces have been designed and fabricated over the past decades. Most of these adhesive surfaces usually fall into one of two contradictory categories: reversible but weak adhesion with anisotropic microstructures, or strong but irreversible adhesion with isotropic micropillars. Anisotropic microstructures such as wedges [20-24] and tilted fibers [25-29] can lead to directional adhesion and friction by shearing in the opposite directions. The structural deformation induced by shearing along the lateral direction contributes to uniform frictional stress, while restricting the adhesion limit in the normal direction owing to a tendency to peel [22-24]. Isotropic micropillars with larger tips [30-33] and composite structures [34, 35] exhibit high adhesion strengths of over $100 \mathrm{kPa}$ or even $\sim 1 \mathrm{MPa}$ by homogenizing the normal interfacial stress. However, equal load sharing among these pillars also prevents them from being easily released. By considering the peeling deformation in gecko toe detachment, various climbing robots [36-42], transport grippers [43-51], and other functional devices [52-55] have been designed based on external field triggered reversible adhesion, such as mechanical [36-44, 52, 53], pressure [46, 47, 51], magnetic [45], thermal [48, 49], and light [50] stimuli. Nonetheless, despite the high adhesion strength of single adhesive microstructures, these existing controllable adhesive systems are limited to smallarea $\left(<5 \mathrm{~cm}^{2}\right)$ surfaces and a lightweight $(<500 \mathrm{~g})$ adhesion capacity, owing to the dilemma of supporting structures simultaneously realizing stiff backing and flexible peeling, particularly in large areas, thereby blocking the broad applications of reversible strong adhesion.

Refocusing on the gecko from a macroscopic perspective, its reversible adhesion also strongly relies on the gripping in and rolling out motions of the toes. The gripping not only aligns the spatula contacts but also stimulates a rigid backing from the tendon with a small angle pulling to achieve a uniform loading, whereas the rolling out forms a sequential peeling of setae with a large angle to realize an easy detachment $[11,56,57]$. In typical sphere-flat adhesive contact tests, a significant change in the adhesion force is observed by matching different stiffnesses of the testing objects and supporting cantilevers [58]. These phenomena indicate that the actual adhesion behavior and resulting adhesion forces are the dual contributions of microscopic intrinsic adhesive interactions and macroscopic controllable loading behaviors. By rationally designing the supporting structures of an adhesive system, the loading behavior can be controlled to achieve the desired adhesion capacity. For example, superglue and pressure-sensitive adhesives (PSAs) with stiff backing can promise a strong adhesion of $100 \mathrm{kPa}-10 \mathrm{MPa}$; however, such adhesives are not reversible upon a mechanical stimulus [59]. With a tendon-enhanced load-sharing design, hand-sized anisotropic adhesive surfaces enable a $70 \mathrm{~kg}$ adult to climb a vertical glass wall with a reversible friction strength of $\sim 100 \mathrm{kPa}$ [60]. Similarly, a fabric-embedded smooth adhesive design also exhibits a high friction capacity by improving the equal load sharing with a high in-plane stiffness [61-63]. Recently, a novel pressure-tuned load-sharing technique enabled a $\sim 20 \mathrm{kPa}$ controllable adhesion for curved substrates [47]. However, a universal and effective strategy to achieve a reversible strong adhesion with controllable loading behavior in a scalable manner remains a significant challenge.

By combining the strong adhesive interactions at the interface and the controllable loading behaviors from the back, we report an extremely simple but effective design, namely, a peeling-based sandwiched composite unit (PSCU), to simultaneously realize stiff-backing strengthened pulling and flexible sequential peeling for a reversible strong adhesion with a scalable performance. The experimental results show that this design can produce a strong adhesion by preventing crack propagation through equal load sharing. Conversely, it can be easily released by causing a stress concentration through sequential edge peeling. These results and the underlying mechanisms were validated through theoretical modeling and a finite element analysis. Furthermore, this composite design was successfully integrated into versatile grippers, inverted ceiling-walking robots, and even $70 \mathrm{~kg}$ adult ceiling-climbing grippers, exhibiting an easily scalable performance without losing the high adhesion strength. This study proposed a universal controllable load sharing design for reversible strong adhesion and demonstrated its promising potential 
in applicable technologies demanding tunable interfacial behaviors.

\section{Experimental}

\subsection{Fabrication of PSCU}

All adhesive layers used in the experiments without extra specifications are mushroom-shaped dry adhesive surfaces made of polyvinyl siloxane [30]. The mushroom-shaped pillars contain a bottom cylindrical stalk with a $40 \mu \mathrm{m}$ diameter and an $80 \mu \mathrm{m}$ height, and a top circular plate with a $2 \mu \mathrm{m}$ thickness and a $45 \mu \mathrm{m}$ diameter, distributed in a hexagonal manner with a center distance of $60 \mu \mathrm{m}$. The measured average surface roughness of the mushroom-shaped cap was $\sim 25 \mathrm{~nm}$ using a 3D optical surface profiler (ZYGO Nexview, USA). A foam block (Xinyunjie Materials Co., Ltd., China) with an optimal hardness of 35 Shore $C$ was selected and fabricated using laser cutting. The steel sheets were fabricated through wire electrode cutting using $65 \mathrm{Mn}$ steel (Quanrui Metal Materials Co., Ltd., China). To enhance the bonding strength between the steel sheet and the adhesive layer, the steel sheet was inked into a prime coat (PR-1200 RTV Prime, Dow Corning, USA), followed by drying for $1 \mathrm{~h}$. It was then coated with a $\sim 100 \mu \mathrm{m}$ thick silicon adhesive film (Sil-Poxy, Smooth-On Inc., USA) on a film coater (TC-100, Mitsui Electric Seiki Co., Ltd., Japan). The adhesive layer was rolled carefully on the silicon adhesive film from one side to the other to avoid any roughness caused by bubbles. After the silicon adhesive film was cured at room temperature, an $\sim 80 \mu \mathrm{m}$ thick epoxy adhesive film (DP 420, 3M Co., Ltd., USA) was created on the other side of the steel sheet using a film coater. The foam was placed on the epoxy adhesive film, forming a strong bond after curing at room temperature (Fig. S1 in the Electronic Supplementary Material (ESM)).

\subsection{Characterization of adhesion/peeling properties of PSCU}

The adhesion and peeling properties of the PSCU were characterized using a homemade apparatus (Fig. S2 in the ESM). Microscopes and goniometers were used in both the $x$ - and $y$-orientations to ensure alignment between the adhesive samples and the substrate (clean glass surface). Two cantilever strain sensors were used to measure the normal and lateral forces. In the adhesion test, the typical load-pull mode was adopted. In particular, the steel sheet maintained its natural state for testing the gripping force while it was bent to the specified peeling distance to test the releasing force. In the peeling test, the preload is implemented with the corresponding weight, and the steel sheet is then bent by pulling the releasing tendon. All experiments were conducted using the same approaching and retracting velocity of $0.1 \mathrm{~mm} / \mathrm{s}$ to minimize viscoelasticity effects on the adhesion. The measured data were sampled with a USB-6002 DAQ module (National Instruments, USA) at 1,000 Hz. The back-pulling and edge-peeling processes under a microscope (BX60, OLYMPUS, Japan) were recorded using a camera (EOS700D, Canon, Japan) at $25 \mathrm{~Hz}$.

\subsection{Numerical simulation}

A finite element analysis was applied using COMSOL (COMSOL Inc., Burlington, MA, USA). The parameters of the PSCU used in the study were obtained from its real geometric dimensions and mechanical properties. To contrast the interfacial stress distributions in the back-pulling and edge-peeling modes, we used the cohesive zone model (CZM) to simulate the adhesive interactions between the adhesive layer and the substrate. The contact pressure was formulated using a penalty method, and the adhesive layer was defined as the objective boundary of the contact. In the study of the effects of steel sheets on the interfacial adhesion state, we adopted a simplified 2D planestrain model to analyze the tensile stress distributions of the micropillars. All ends of the micropillars were assigned a fixed constraint to emulate the frictional adhesion at the interface. The backing side was applied with a homogeneous normal stress of $100 \mathrm{kPa}$ as the force boundary.

\subsection{Fabrication of grippers and robots}

a) A four-legged gripper for picking and placing both flat and curved objects: The self-adaptive-locking mechanism (SALM) in the length direction is composed of a linear bearing, a slide bar with a through hole, a return spring, and a clamp. The clamps can be 
actuated by a servo motor to lock the length position, whereas the spring can ensure that it returns to its initial position. The other SALM in the angular direction consists of a rough and separable rotation pair, a return spring, and a slide bar. When the bar is pulled up through the hole, the spring is compressed and the rotation pair is separated, allowing the PSCU to rotate around its center freely. By contrast, when the bar is released, the spring returns to its initial position and compresses the rough contact pair, the frictional torque of which ensures the locking of the angular position. The four adhesive legs are aligned in a square-diagonal layout, through which all PSCUs can be peeled off from the center with only one servo motor. In addition, this layout can adapt to object surfaces of different shapes. When all four PSCUs are horizontal, they can grasp flat objects; when the opposite two are angled while the other two are horizontal, it can handle cylindrical objects with tangential adhesion, and when all four PSCUs are angled, they can grasp spherical objects with tangential adhesion.

b) A ceiling-walking robot: Each modularized adhesive leg has three PSCUs fixed on the leg base in a triangular arrangement. A spherical hinge (GE4C, NSK Ltd., Japan) and three return springs were used between the base and the leg to increase the adaptability of the adhesive leg and compensate for the unflatness among the four legs. Linear guides (Hiwin Technologies Corp., China) were used for up-and-down and forward motions, and slider-crank mechanisms with a servo motor were adopted for their actuation. To increase the stiffness to weight ratio, the robot frame was engraved with a carbon fiber reinforced plate (Shanmeibang Carbon Fiber Material Co., Ltd., China). Servo motors and batteries were purchased from Yinyan Model Tech, Ltd., China, whereas the control board was homemade. The connecting parts were fabricated with a photosensitive resin (DSM, the Netherlands) using a 3D printer (Form 2, Formlabs Inc., USA).

\section{Results and discussion}

\subsection{Design and basic mechanisms of the PSCU}

As shown in Figs. 1(a) and 1(b), the geckos switch
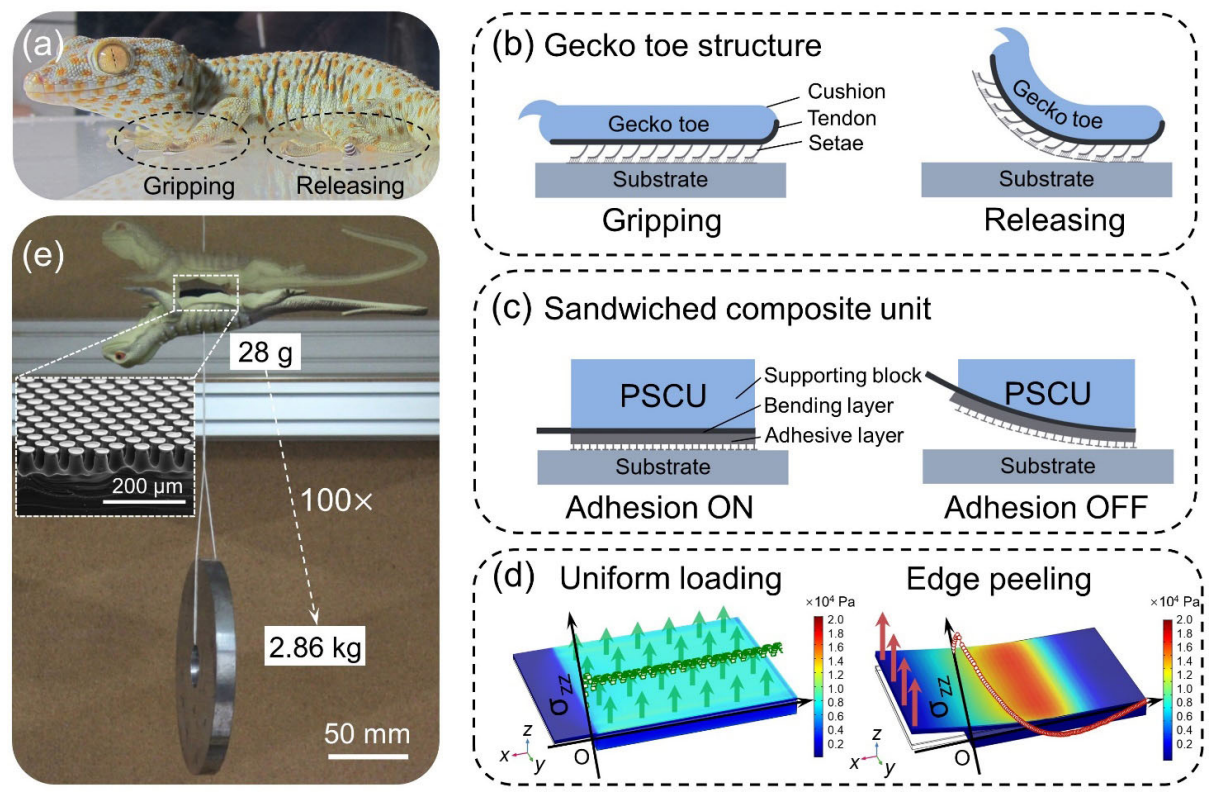

Fig. 1 Design of the PSCU. (a) A gecko running on a glass surface with two toes in a gripping state while the other two toes are in a releasing state. (b) Schematic illustration of the gripping and releasing states of gecko toe structure, composed of cushion, tendon, and setae. (c) Schematic illustration of the proposed PSCU to switch on/off adhesion. (d) Normal stress distribution of the adhesive interface and Von Mises stress distribution of the steel sheet and the adhesive layer for uniform loading and edge peeling, respectively. The green arrow represents the adhesion force, whereas the red arrow represents the peeling force. (e) A gecko-inspired controllable gripping device loading a $2.86 \mathrm{~kg}$ mass on a glass ceiling with a $28 \mathrm{~g}$ self-weight. The inset shows the scanning electron microscope image of the mushroom-shaped dry adhesive surface. 
their toes from a gripping state to a releasing state by peeling, based on a tendon-actuated bending motion. The core structure of a gecko toe can be simplified as shown in the schematic in Fig. 1(b), and is composed of a soft cushion, embedded tendon, and adhesive setae [24, 61, 63]. The adhesive setae provide microscopic adhesive interactions at the interface, supported by the soft cushion and embedded tendon. Geckos grip the substrate firmly by pulling the tendon to transmit the interfacial stress to their skeleton for attachment, while allowing the tendon and muscle to bend and peel the toe pad from the substrate for detachment. Consequently, geckos can achieve reversible adhesion by actuating/recovering the bending deformation of the toes. Inspired by the composite structure and the transition mechanism, we proposed a PSCU to realize switchable strong adhesion for artificial adhesive surfaces, as shown in Fig. 1(c). In this design, a bending layer is sandwiched between an adhesive layer at the bottom and a supporting block at the top, connected to an actuator such as a motorized stage and a robotic arm from the backside. The adhesive layer works like the adhesive setae of a gecko toe, which provides strong adhesive interactions through intrinsic van der Waals forces at the contact interface, such as commercial adhesive tape and a popular mushroom-shaped adhesive surface. The bending layer works as an anti-stretching backing similar to the embedded tendon of the gecko toe to support the adhesive load uniformly for attachment in the natural state; however, the adhesive layer is peeled at a large peeling angle to decrease the adhesive area sharply for detachment in the bending state. The supporting block has the same function as the cushion and muscle of the gecko toe, which not only transmits tensile stress for adhesion but also reversibly deforms for peeling. Therefore, the PSCU can yield a strong attachment (Adhesion $\mathrm{ON}$ ) with proper preload enabled intimate contact and easy detachment (Adhesion OFF) after peeling from the substrate, as shown in Fig. 1(c). By dynamically bending and recovering the bending layer, the PSCU can reversibly change between the states of Adhesion $\mathrm{ON}$ and Adhesion OFF, thereby achieving a reversible strong adhesion. Considering the mature and stable fabrication and reusability of the mushroom-shaped adhesive surface, it was used as the adhesive layer in our experiments. A steel sheet and soft foam were chosen as the bending layer and the supporting block, respectively, owing to their low cost and easy accessibility.

A basic finite element analysis (FEA) based on the CZM was applied to examine the adhesive stress distribution of the PSCU during attachment and detachment. As shown in Fig. 1(d), the bending layer provides a uniform loading at the adhesive interface, which can efficiently utilize the adhesive stresses of the entire contact area and achieve a strong adhesion. In the peeling state, a tensile stress concentration occurs at the macroscopic peel front, whereas a compressive stress exists in the remaining adhesive area, indicating an easy peeling process and subsequent detachment. The FEA simulation provides basic explanations for the reversible working mechanism of the PSCU. As a simple demonstration, combined with mushroom-shaped dry adhesive surfaces, this switchable strong adhesion enables a "gecko toe" to hold and release an object with two orders of magnitude over its own weight on an inverted glass ceiling surface, as shown in Fig. 1(e).

\subsection{Mechanical performances of the PSCU}

We first investigated the adhesion and peeling behavior of a simplified adhesive unit composed of an adhesive layer and a bending layer. The mushroom-shaped adhesive surface, $15 \mathrm{~mm}$ in length and $10 \mathrm{~mm}$ in width, was bonded on a $150 \mu \mathrm{m}$ thick steel sheet as the sample. Figures 2(a) and 2(b) show the typical force measurements and in situ loading states of the adhesive unit for adhesion (back-pulling mode) and peeling (edge-peeling mode), respectively. The adhesive unit approaches a glass substrate until the preload reaches $1.0 \mathrm{~N}$, followed by retraction from its back and edge. In situ loading states were captured during the retracting process using the inserted microscopic images. They showed that the peel zone of an individual pillar (marked with a red circle) during the adhesion test covers almost the entire adhesive interface, whereas that during the peeling test covers only the stretched area at the macroscopic peel front (the dashed line area in Fig. 2(b)) [64, 65], corresponding to the adhesive stress distribution in Fig. 1(d) (see Figs. S3 and S4 and Vedios S1 and S2 in the ESM for 
(a)

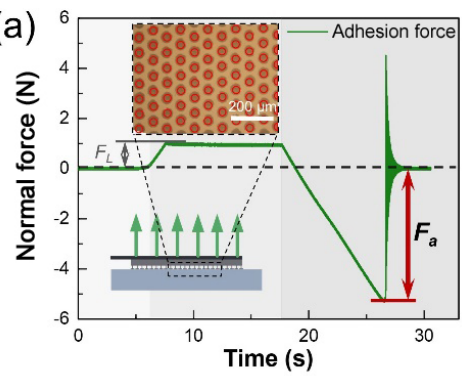

(d)

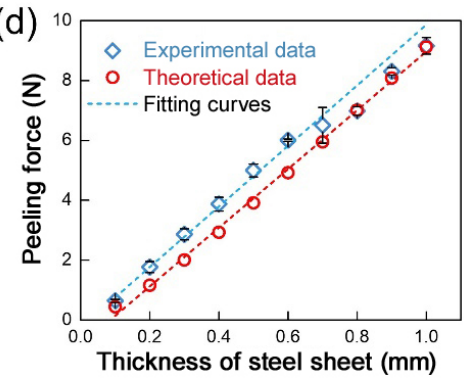

(b)

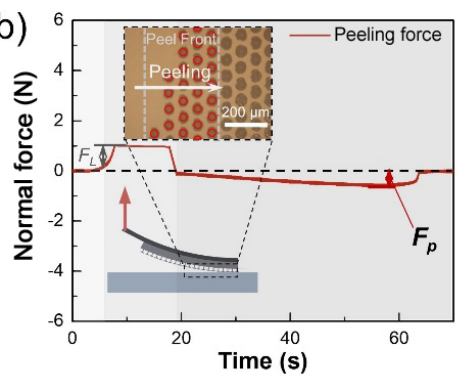

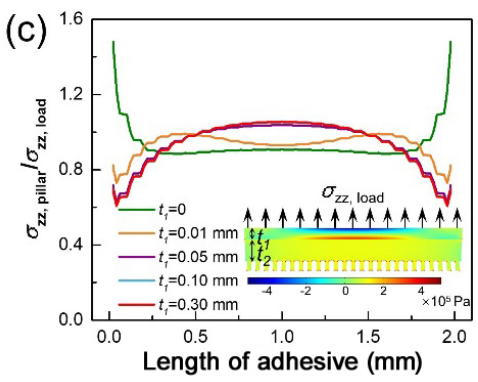

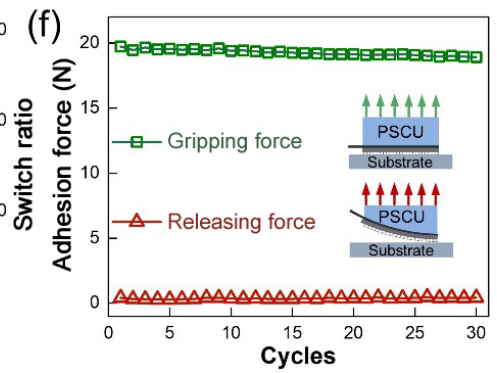

Fig. 2 Mechanical performance characterization and analysis of the PSCU. (a) Representative force versus time curves for adhesion force in back-pulling mode and (b) peeling force in edge-peeling mode. The schematic insets represent the corresponding loading states of the adhesive unit from the side view. The microscopic images show corresponding detaching states of the micropillars from glass substrates, where the peel zones (microscopic crack front) of individual stretched pillars are highlighted with red circles. (c) 2D plane-strain simulation results of tensile stress distribution in pillars along the length direction of the adhesive layer for different steel sheet thicknesses. The inset represents the corresponding simulation image of the applied stress and normal stress distribution in the steel sheet and adhesive layer. The data were taken from the nodes at the middle of the pillars and normalized by dividing the local stress by the applied homogeneous normal stress. (d) Relationship between the peeling force of the adhesive surface and the thickness of the steel sheet for both experimental and theoretical results. (e) Adhesion force and switch ratio of the PSCU versus different peeling distances of the steel sheet. The schematic inset presents the corresponding state of the PSCU from the side view. (f) Repeatability tests of gripping forces and releasing forces for the optimized PSCU.

more details). Consequently, the measured adhesion force $F_{\mathrm{a}}$ reaches up to $5.3 \mathrm{~N}$, whereas the peeling force $F_{\mathrm{p}}$ is only $0.6 \mathrm{~N}$. The basic tests indicate that this design can provide strong adhesion for attachment while be easily peeled off for detachment.

The effects of the design parameters on the adhesion and peeling performances of the simplified adhesive unit were then systematically investigated. We conducted an FE simulation based on a simplified plane-strain model to analyze the underlying effects of the bending layer on the adhesion capacity. As shown in Fig. 2(c), the direct loading of the elastomeric mushroom-shaped adhesive surface with a certain backing thickness $\left(t_{2}=0.2 \mathrm{~mm}\right)$ cannot avoid the edge stress concentration, which can easily cause crack propagation and a consequent low adhesion force. By contrast, a more uniform tensile stress distribution can be achieved by adding a bending layer such as a steel sheet with a high elastic modulus $\left(E_{1}=200 \mathrm{GPa}\right)$ and a certain thickness $\left(t_{1}=0.1 \mathrm{~mm}\right)$, contributing to an enhanced adhesion. In a typical fibrillar adhesive design, discrete micropillars are designed to prevent a lateral stress transmission and consequent crack propagation. However, these micropillars must be connected by a backing membrane with a certain thickness when applying existing elastomer fabrication techniques. A detailed deformation analysis of this system reveals that the backing membrane generates lateral shrinkage at the edge, similar to the classic flat/punch adhesive contact problem. By contrast, a bending layer made of a rigid material can constrain the lateral shrinkage deformation to homogenize the normal stress distribution (Fig. S5 in the ESM), and this effect will be saturated when its thickness is more than $0.1 \mathrm{~mm}$ (Fig. 2(c)). It was also proved that the bending layer homogenization effect only works when the elastic modulus of the bending layer is at a gigapascal scale, that is, larger than that of the adhesive layer by over three orders of magnitude (Fig. S6(a) in the ESM). Most importantly, we found that the adhesive 
stress can reach the most uniform state by connecting the pillars directly to the steel sheet without any soft backing membrane $\left(t_{1}=0\right)$, thereby generating no lateral shrinkage for stress singularity at the contact edge. Despite the challenges in practical fabrication techniques, a thinner adhesive layer is always beneficial for a more uniform load sharing (Fig. S6(b) in the ESM), in agreement with previous experimental results [66]. We also investigated the effects of the adhesive surface size and substrate radius on the adhesion performance of PSCU (Fig. S7 in the ESM), providing basic guidelines for an optimal design. We found that a larger sample size can lead to a smaller adhesion strength on a flat substrate with the same preload, which may be caused by the poor contact condition and misalignment for larger samples. For curved substrates, the adhesion force decreases as the substrate curvature increases, which is caused by uneven contact and loading conditions. For substrates with higher curvatures, the flat sample has poor contact with only a small area owing to the finite compressive deformation. Meanwhile, the central position tends to be compressive, whereas the surrounding position tends to be tensile in the loading process, which further decreases the adhesion force.

To estimate the peeling force and achieve an easy peeling, we established an analytical model for the edge-peeling mode based on the principle of minimum potential energy (see Fig. S8 and the corresponding text in the ESM for more details). In this model, the bending deformation and external peeling force can be solved for a given peeling distance, which can reveal the peeling behavior of the simplified adhesive unit. By varying the thickness of the bending layer, we can obtain the peel zone length and the corresponding peeling force, as shown in Fig. S8(b) in the ESM. It is obvious that both the peel zone length and the peeling force increase almost linearly with the thickness of the steel sheet. It was found that before the peeling distance reached the maximum value, the peel zone length remained nearly constant for the same steel sheet. More generally, the adhesive layer with a lower bending stiffness of the backing tends to generate a smaller peel zone length when pulled from the edge, which would result in a lower peeling force. However, as Fig. S8(c) in the ESM shows, a lower back bending stiffness also leads to a larger peeling distance, which can increase the size of the adhesive unit. We also tested the peeling force of the simplified adhesive unit for different bending layer thicknesses. As shown in Fig. 2(d), the experimental results are in good agreement with the theoretical results, particularly for the specific peeling force and the linearly increasing tendency. In addition, a larger length/width ratio of the steel sheet would benefit its peeling for detachment with a lower bending stiffness (Fig. S9 in the ESM). In addition, the peeling velocity under normal conditions only has a minor influence on the peeling force for steel sheets with a thickness of less than $0.4 \mathrm{~mm}$ (Fig. S10 in the ESM).

Based on these results, the adhesive layer should have a small thickness to eliminate the lateral shrinkage and consequent edge stress concentration for strong adhesive interactions at the interface. The bending layer should be designed rationally by considering the strong adhesion, low peeling force, and compact structural design, yielding a controllable strong loading behavior from the back. Therefore, the bending layer should have a high elastic modulus and a proper thickness to simultaneously enhance the adhesion and facilitate the peeling. The supporting block should be anti-stretched to transmit a uniform stress from the bending layer and easily compressible to facilitate complete peeling. In other words, the supporting block has an optimal stiffness by considering both strong adhesion and easy peeling. In addition, it should have a thickness larger than the maximum peeling distance of the adhesive layer to ensure complete peeling.

Given the design criteria above, an optimal PSCU was designed with a $100 \mu \mathrm{m}$ thick adhesive layer, a $100 \mu \mathrm{m}$ thick steel sheet, and an $8 \mathrm{~mm}$ thick foam block. To examine the performances of strong attachment and easy detachment, we investigated the influence of the pre-implemented peeling distance $(S)$ on the adhesion force and switch ratio, as shown in Fig. 2(e). The peeling distance was implemented after the applied preload, followed by retraction from the back. When $S=0$, the PSCU reaches the attachment state; in addition, when $S>0$, it switches to detachment states with different pre-peeling levels. The switch ratio is obtained by dividing the measured adhesion 
force of $S=0$ by that of $S>0$. The adhesion force decreases severely as the peeling distance increases owing to the decreased contact area and increased peel angle $[9,12]$. The gripping force $(S=0)$ of the optimized PSCU was approximately $18.9 \mathrm{~N}$ (with an adhesion strength of $\sim 120 \mathrm{kPa}$ ), whereas its releasing force $(S=5 \mathrm{~mm})$ is $0.35 \mathrm{~N}$, reaching a high switch ratio of approximately 54 . The repeatability of its switching capability was tested over 30 cycles, exhibiting a relatively stable performance, as shown in Fig. 2(f). To validate the general switching ability of most adhesive layers, we measured the gripping and releasing forces for two types of mushroom-shaped adhesive surfaces and two types of commercial adhesive tapes (Fig. S11 in the ESM). It is proved that the PSCU not only works well for microstructured adhesive surfaces but also switches the adhesion of unstructured adhesives such as PSAs, which extends the application range of this design.

\subsection{Practical application demonstrations}

A four-legged gripper was developed based on the PSCU, which can handle both flat and curved objects.
Figures 3(a) and 3(b) show the design and fabrication of the gripper integrated with four PSCUs. Two SALMs were designed for each PSCU in both the length and angular directions to increase its adaptability to the target surfaces with differences in height and curvature, thereby reliably handling both flat and curved surfaces. Figure 3(c) shows a typical working mechanism for gripping and releasing curved objects in four steps, which is similar to flat object manipulation. In the first step of approaching the object surface, the two SALMs are set free by a motor to adapt to the surface topography. They are then locked to maintain the adapted contact state, enabling equal load sharing among the four PSCUs to achieve a high load capacity. The releasing tendons are pulled up by actuating another motor to pre-peel the adhesive layer from the substrate such that the gripper can be easily released from the object.

This versatile gripper can handle the flat surfaces of a book and a power supply, as well as curved surfaces of a beaker and football (Figs. 3(d)-3(g) and Vedio S3 in the ESM), showing a dexterous grasping ability. As shown in Vedio S4 in the ESM, a

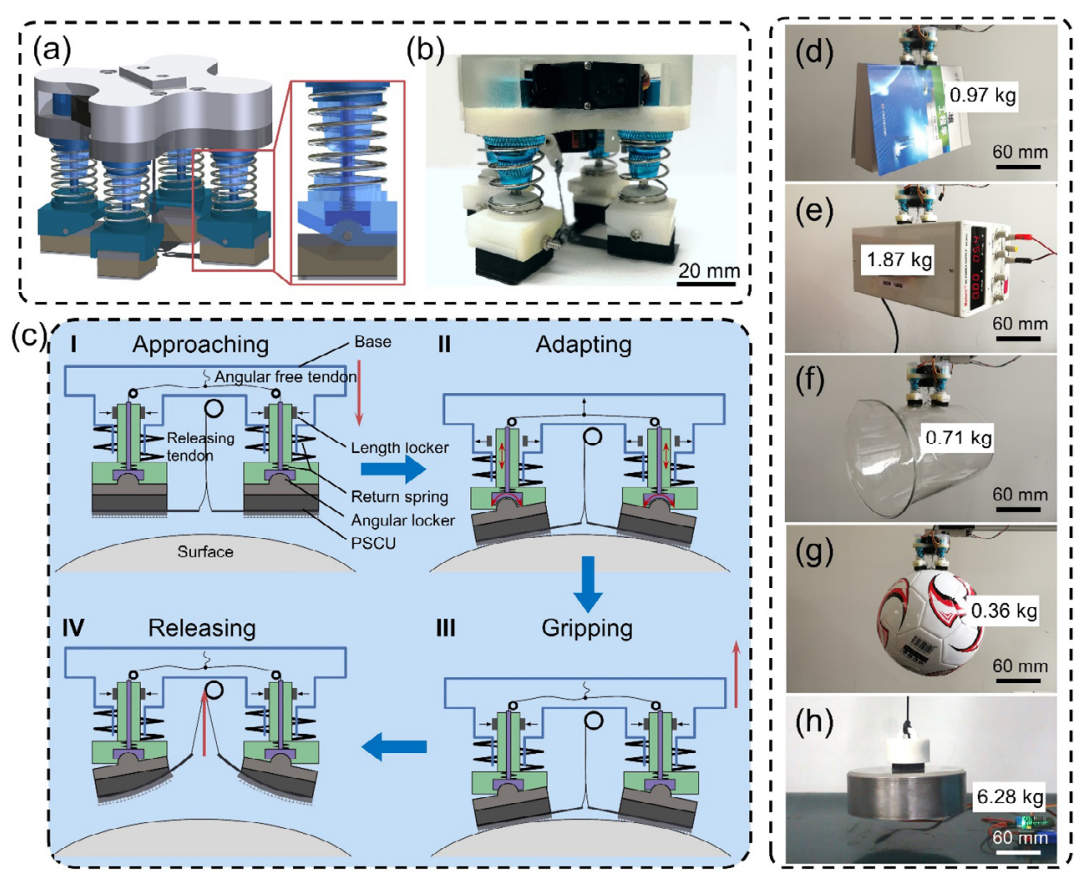

Fig. 3 Demonstration of transferring grippers. (a) The CAD model and (b) fabricated gecko-inspired four-legged gripper for both flat and curved surface grasping. The inset represents two self-adaptive-locking mechanisms. (c) The proposed working mechanism of the gripper for a curved surface. The developed gecko-inspired gripper can grasp various kinds of 3D objects such as (d) a $0.97 \mathrm{~kg}$ book, (e) an $1.87 \mathrm{~kg}$ DC power supply, (f) a $0.71 \mathrm{~kg}$ beaker, and (g) a $0.36 \mathrm{~kg}$ football. (h) An integrated gripper with a $103 \mathrm{~g}$ self-weight can grasp a $6.28 \mathrm{~kg}$ flat mass without applying an external preload or alignment. 
manipulation process for flat objects was successfully demonstrated using a six-axis robot (ABB, IRB 120, Sweden). The $3.2 \mathrm{~kg}$ flat mass can be stably gripped, transferred, and released. Furthermore, we developed a novel integrated gripper based on the PSCU with a self-weight of $103 \mathrm{~g}$ and an adhesive area of $8.4 \mathrm{~cm}^{2}$. It can adhere to a $6.28 \mathrm{~kg}$ flat surface without applying any external preload or alignment for engagement, and detach from it easily, as shown in Fig. 3(h) and Vedio S5 in the ESM.

As another possible demonstration of the PSCU, we developed an inverted ceiling-walking robot with a weight of approximately $320 \mathrm{~g}$ by mimicking gecko climbing [11-13]. This four-legged robot consists of two robot frames, each of which is fixed with two diagonal adhesive legs. All adhesive legs are modularized and independent and can be replaced quickly in case of contamination or damage. There are two servo motors on each leg: one for up-anddown movement and the other for switching adhesion. Another servo motor enables the forward locomotion of the robot, during which one robot frame remains attached while the other in detachment moves forward. This locomotion can be repeated in six phases to realize independent and continuous walking without any sophisticated feedback, as shown in Figs. 4(a)-4(f) and Vedio 56 in the ESM.

To further demonstrate the exceptional performance of the PSCU, we scaled it up to a large area of approximately $154 \mathrm{~cm}^{2}$ for human climbing. We adopted contact splitting principles to ensure a compact structure and compliant engagements by arranging these PSCUs in a hexagonal distribution. As shown in Figs. 5(a) and 5(b), the human climbing gripper was divided into 108 triangular PSCUs, each with an area of $1.4 \mathrm{~cm}^{2}$. There are six servo motors in total, each with a maximal torque of $0.34 \mathrm{~N} \cdot \mathrm{m}$ to pull the releasing tendons of the 18 PSCUs. A spring-bolt locker was designed at the end of the handle for equal loading and avoiding any misalignment between the two loading plates. With this hand-sized climbing gripper, we achieved a stable grip and easy release
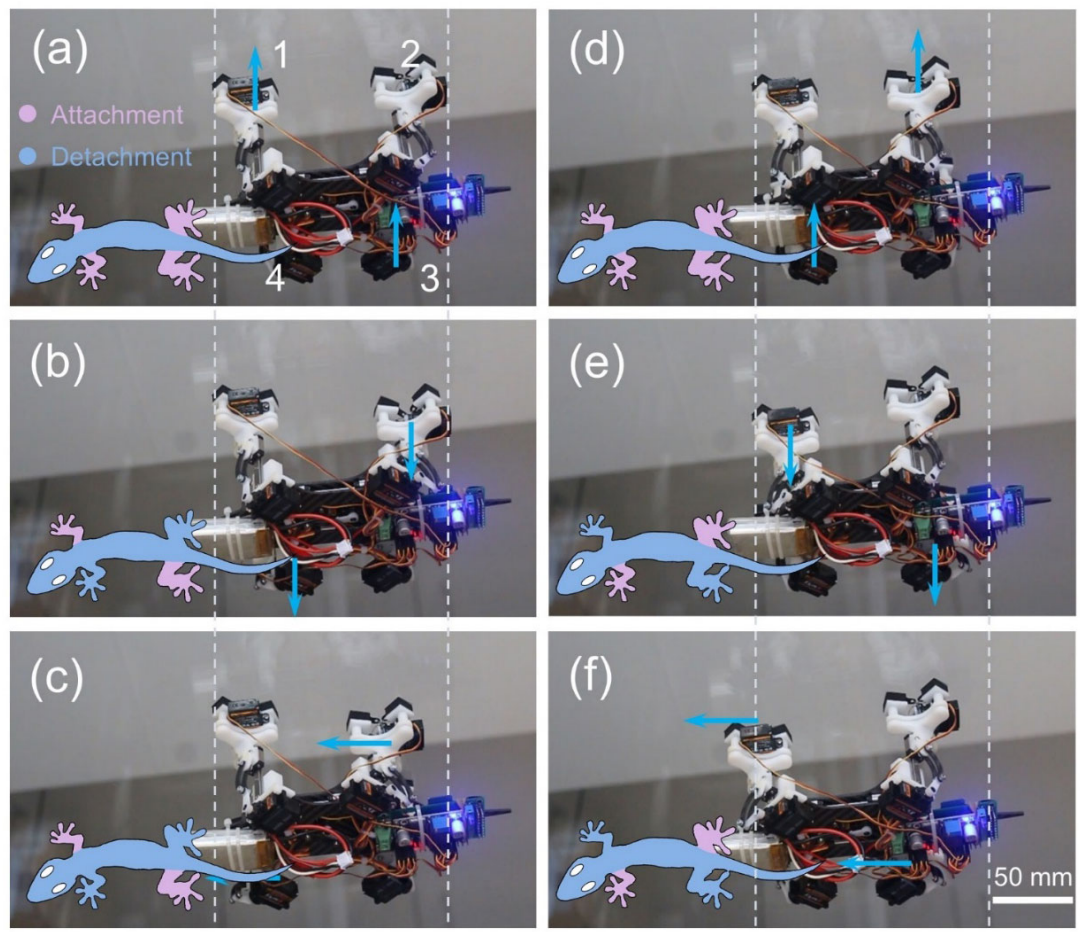

Fig. 4 Demonstration of gecko-inspired four-legged robot climbing on an inverted glass ceiling. (a) Four legs all attached on the ceiling. (b) Legs 2 and 4 peeled from the ceiling with the other two legs attached to the ceiling. (c) The half robot body with legs 2 and 4 moving forward in the lateral direction. (d) Legs 2 and 4 attached to the ceiling. (e) Legs 1 and 3 peeled from the ceiling, while the other two are attached to the ceiling. (f) The other half robot body with legs 1 and 3 moving forward in the lateral direction. The gecko inset represents the gait of the robot, in which the purple color represents the attached state and the blue color represents the detached state. The white scale bar indicates $50 \mathrm{~mm}$ in length. 

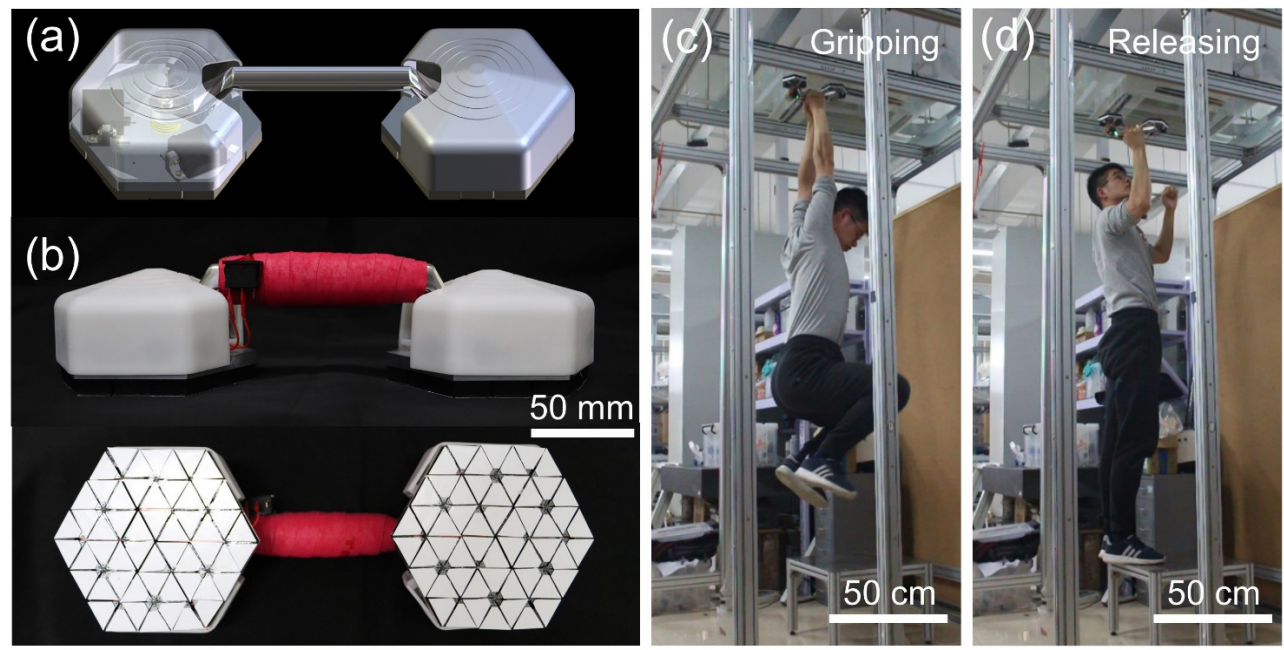

Fig. 5 Design and demonstration of human climbing grippers. (a) The CAD model and (b) fabricated human climbing gripper. (c) The human climbing gripper stably holds a $70 \mathrm{~kg}$ adult and (d) is easily released.

for a $70 \mathrm{~kg}$ adult, as shown in Figs. 5(c) and 5(d). Details of this gripping and releasing demonstration are provided in Vedio S7 in the ESM. To the best of our knowledge, bio-inspired reversible adhesion can be utilized for human beings climbing on ceilings. It is worth mentioning that owing to the intrinsic van der Walls interactions, this compact scalable design has the advantages of a small volume, light weight, low energy consumption, and portable features in contrast to traditional suction techniques, with a great potential for grasping and manipulation tasks in the space environment.

To demonstrate the reversible strong adhesion of our scalable composite design, we contrast the adhesion load capacity and the corresponding adhesive area with previous designs of adhesives and devices. As shown in Fig. 6, our PSCU exhibits a positive sizescaling slope across a wide range of adhesive areas of over two orders of magnitude. Compared with geckos and previous studies on switchable dry adhesive surfaces and devices, the maximal adhesion force of PSCU-based devices is almost one order of magnitude higher for the same adhesive area. This capacity is attributed to the dual functions of the bending layer, that is, uniform loading in the natural state for attachment and flexible peeling in the bending state for detachment. Its working mechanism of enhanced normal adhesion is the same as the in-plane stiffness for uniform lateral friction, that is, by restraining the lateral shrinkage caused stress concentration in the adhesive layer [60-63]. It is interesting to note that this effect is essentially similar to the concept of contact splitting. To prevent the evolution of edge stress singularity, the contact splitting principle proposes discrete or micropatterned structure arrays near the interface $[4,16]$. Here, we show that the stiffness of the backing that connects the discrete structures is also important for eliminating the edge stress concentration. This design can also utilize a bending deformation actuated edge stress to peel the adhesive layer for easy detachment. Therefore, the two contradictory states of uniform loading and flexible peeling can be united in our design by utilizing the

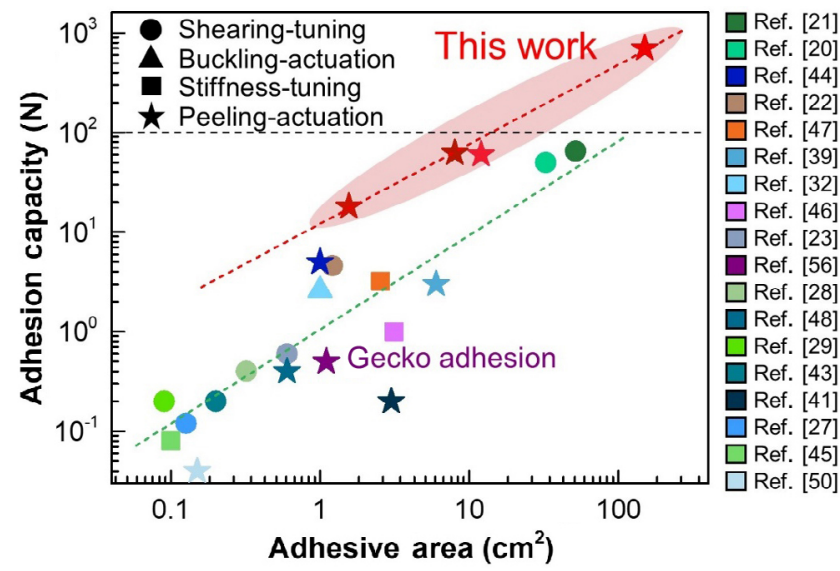

Fig. 6 Adhesion capacity scaling of the proposed PSCU and comparison with previously reported controllable dry adhesive surfaces and devices. The reported controllable dry adhesion was classified with different marked shapes according to specific modulation method applied [17]. 
natural stretching state and the active bending state of the sandwiched bending layer, fully exploiting the adhesion strength of various adhesive layers for a reversible strong adhesion.

Geckos possess remarkable reversible adhesion and friction for locomotion; nonetheless, the L configuration and limited stiffness of the toe structures determine that their actual adhesion strength at the body level is relatively low ( $20 \mathrm{kPa})$ [56]. Focusing on the cross-scale material structures spanning from microscale setae to macroscale tendons and the peeling motion principles of gecko toes, we developed a novel sandwiched composite design with consistent controllable adhesion performances at different size scales. The soft-hardsoft composite design meets the demands of full and intimate contact of most pillars before loading, and achieves a uniform interfacial stress distribution among the pillars for maximal loading capacity. Therefore, it can still achieve an adhesion strength of $\sim 100 \mathrm{kPa}$ in an area of dozens of square centimeters, thus providing a high load capacity for related devices (Fig. 6). It can also flexibly bend the bending layer to peel the adhesive layer rapidly without stretching the layer, contributing to the reversible and repeatable performance of this design. As a result, it can transform from an adhesion state into to detachment state within $\sim 0.2 \mathrm{~s}$ (response time of the peeling actuation) with extremely small reactive forces, providing both stability and mobility for functional devices (Fig. 2(f)). With the development of fabricated adhesives, we believe that the combination of this macroscopic backing design and microscopic adhesive structures can be engineered for broader applications demanding reversible strong adhesion.

\section{Conclusions}

In summary, we presented a sandwiched composite design to realize strong adhesion and easy detachment for adhesive surfaces by mechanically tuning the interfacial stress distribution. The design exhibits a stable, repeatable, and reversible strong adhesion, with an easily scalable capacity for a large area ranging from $\sim 1.5$ to $150 \mathrm{~cm}^{2}$ and a high load spanning from $\sim 20$ to $700 \mathrm{~N}$. This biomimetic design provides a generally applicable solution for the switchable strong adhesion of various adhesive surfaces. We demonstrated versatile grippers handling both flat and curved objects and ceiling-waking robots walking on an inverted ceiling. Moreover, a climbing gripper for an adult was successfully implemented to fulfill the gecko-like movement for human beings. This controllable load sharing strategy provides a paradigm for the implementation of reversible strong adhesion and the design of applicable devices in medical, industrial, space, and other relevant fields in demand.

\section{Acknowledgements}

This work was supported by the National Natural Science Foundation of China (Grant No. 51425502). We dedicate this article to our great advisor, colleague, and dear friend Jacob N. ISRAELACHVILI, who passed away before we finish this work. We gratefully thank him for his contribution and guidance to the research of surface and interface, long-time collaboration, and guidance for us in the field of intermolecular and surface forces. Thanks to Prof. Jinyou SHAO at the State Key Laboratory for Manufacturing Systems Engineering of Xi'an Jiaotong University, China, for providing us some mushroom-shaped dry adhesive surface samples.

Electronic Supplementary Material Supplementary material is available in the online version of this article at https://doi.org/10.1007/s40544-021-0522-4.

Open Access This article is licensed under a Creative Commons Attribution 4.0 International License, which permits use, sharing, adaptation, distribution and reproduction in any medium or format, as long as you give appropriate credit to the original author(s) and the source, provide a link to the Creative Commons licence, and indicate if changes were made.

The images or other third party material in this article are included in the article's Creative Commons licence, unless indicated otherwise in a credit line to the material. If material is not included in the article's Creative Commons licence and your intended use is not permitted by statutory regulation or exceeds the permitted use, you will need to obtain permission directly from the copyright holder. 
To view a copy of this licence, visit http://creativecommons.org/licenses/by/4.0/.

\section{References}

[1] Autumn K, Liang Y A, Tonia Hsieh S, Zesch W, Chan W P, Kenny T W, Fearing R, Full R J. Adhesive force of a single gecko foot-hair. Nature 405(6787): 681-685 (2000)

[2] Autumn K, Sitti M, Liang Y A, Peattie A M, Hansen W R, Sponberg S, Kenny T W, Fearing R, Israelachvili J N, Full $\mathrm{R}$ J. Evidence for van der Waals adhesion in gecko setae. PNAS 99(19): 12252-12256 (2002)

[3] Feshanjerdi M. Electroadhesion between a flat touchscreen and the human finger with randomly self-affine fractal surface. Friction 9(1): 132-142 (2021)

[4] Arzt E, Gorb S, Spolenak R. From micro to nano contacts in biological attachment devices. Proc Natl Acad Sci USA 100(19): 10603-10606 (2003)

[5] Myshkin N, Kovalev A. Adhesion and surface forces in polymer tribology-A review. Friction 6(2): 143-155 (2018)

[6] Meng Y G, Xu J, Jin Z M, Prakash B, Hu Y Z. A review of recent advances in tribology. Friction 8(2): 221-300 (2020)

[7] Gao H, Yao H. Shape insensitive optimal adhesion of nanoscale fibrillar structures. PNAS 101(21): 7851-7856 (2004)

[8] Kendall K. The adhesion and surface energy of elastic solids. J Phys D: Appl Phys 4(8): 1186-1195 (1971)

[9] Kendall K. Thin-film peeling-the elastic term. J Phys D: Appl Phys 8(13): 1449-1452 (1975)

[10] Spuskanyuk A V, McMeeking R M, Deshpande V S, Arzt E. The effect of shape on the adhesion of fibrillar surfaces. Acta Biomater 4(6): 1669-1676 (2008)

[11] Tian Y, Pesika N, Zeng H, Rosenberg K, Zhao B, McGuiggan $\mathrm{P}$, Autumn K, Israelachvili J. Adhesion and friction in gecko toe attachment and detachment. PNAS 103(51): 19320-19325 (2006)

[12] Autumn K, Dittmore A, Santos D, Spenko M, Cutkosky M. Frictional adhesion: A new angle on gecko attachment. J Exp Biol 209(Pt 18): 3569-3579 (2006)

[13] Wang Z Y, Xing Q, Wang W B, Ji A H, Dai Z D. Contribution of friction and adhesion to the reliable attachment of a gecko to smooth inclines. Friction 6(4): 407-419 (2018)

[14] Pesika N S, Tian Y, Zhao B X, Rosenberg K, Zeng H B, McGuiggan P, Autumn K, Israelachvili J N. Peel-zone model of tape peeling based on the gecko adhesive system. $J$ Adhesion 83(4): 383-401 (2007)

[15] Zhou M, Pesika N, Zeng H B, Tian Y, Israelachvili J. Recent advances in gecko adhesion and friction mechanisms and development of gecko-inspired dry adhesive surfaces. Friction 1(2): 114-129 (2013)

[16] Hensel R, Moh K, Arzt E. Engineering micropatterned dry adhesives: From contact theory to handling applications. Adv Funct Mater 28(28): 1800865 (2018)

[17] Li X S, Tao D S, Lu H Y, Bai P P, Liu Z Y, Ma L R, Meng Y G, Tian Y. Recent developments in gecko-inspired dry adhesive surfaces from fabrication to application. Surf Topogr: Metrol Prop 7(2): 023001 (2019)

[18] Croll A B, Hosseini N, Bartlett M D. Switchable adhesives for multifunctional interfaces. Adv Mater Technol 4(8): 1900193 (2019)

[19] Amjadi M, Kyung K U, Park I, Sitti M. Stretchable, skinmountable, and wearable strain sensors and their potential applications: A review. Adv Funct Mater 26(11): 1678-1698 (2016)

[20] Jiang H, Hawkes E W, Arutyunov V, Tims J, Fuller C, King J P, Seubert C, Chang H L, Parness A, Cutkosky M R. Scaling controllable adhesives to grapple floating objects in space. In Proceedings of the 2015 IEEE International Conference on Robotics and Automation (ICRA), Seattle, USA, 2015: 2828-2835.

[21] Jiang H, Hawkes E W, Fuller C, Estrada M A, Suresh S A, Abcouwer N, Han A K, Wang S Q, Ploch C J, Parness A, et al. A robotic device using gecko-inspired adhesives can grasp and manipulate large objects in microgravity. Sci Robot 2(7): eaan4545 (2017)

[22] Day P, Eason E V, Esparza N, Christensen D, Cutkosky M. Microwedge machining for the manufacture of directional dry adhesives. J Micro Nano Manuf 1(1): 011001 (2013)

[23] Tao D S, Gao X, Lu H Y, Liu Z Y, Li Y, Tong H, Pesika N, Meng Y G, Tian Y. Controllable anisotropic dry adhesion in vacuum: Gecko inspired wedged surface fabricated with ultraprecision diamond cutting. Adv Funct Mater 27(22): 1606576 (2017)

[24] Cutkosky M R. Climbing with adhesion: From bioinspiration to biounderstanding. Interface Focus 5(4): 20150015 (2015)

[25] Murphy M P, Aksak B, Sitti M. Gecko-inspired directional and controllable adhesion. Small 5(2): 170-175 (2009)

[26] Jeong H E, Lee J K, Kim H N, Moon S H, Suh K Y. A nontransferring dry adhesive with hierarchical polymer nanohairs. PNAS 106(14): 5639-5644 (2009)

[27] Chary S, Tamelier J, Turner K. A microfabricated geckoinspired controllable and reusable dry adhesive. Smart Mater Struct 22(2): 025013 (2013)

[28] Jin K, Cremaldi J C, Erickson J S, Tian Y, Israelachvili J N, Pesika N S. Biomimetic bidirectional switchable adhesive inspired by the gecko. Adv Funct Mater 24(5): 574-579 (2014) 
[29] Wang Y, Tian H M, Shao J Y, Sameoto D, Li X M, Wang L, $\mathrm{Hu}$ H, Ding Y C, Lu B H. Switchable dry adhesion with step-like micropillars and controllable interfacial contact. ACS Appl Mater Interfaces 8(15): 10029-10037 (2016)

[30] Gorb S, Varenberg M, Peressadko A, Tuma J. Biomimetic mushroom-shaped fibrillar adhesive microstructure. J Royal Soc Interface 4(13): 271-275 (2007)

[31] Wang Y, Hu H, Shao J Y, Ding Y C. Fabrication of welldefined mushroom-shaped structures for biomimetic dry adhesive by conventional photolithography and molding. ACS Appl Mater Interfaces 6(4): 2213-2218 (2014)

[32] Purtov J, Frensemeier M, Kroner E. Switchable adhesion in vacuum using bio-inspired dry adhesives. ACS Appl Mater Interfaces 7(43): 24127-24135 (2015)

[33] Fischer S C L, Groß K, Abad O T, Becker M M, Park E, Hensel R, Arzt E. Funnel-shaped microstructures for strong reversible adhesion. Adv Mater Interfaces 4(20): 1700292 (2017)

[34] Minsky H K, Turner K T. Composite microposts with high dry adhesion strength. ACS Appl Mater Interfaces 9(21): 18322-18327 (2017)

[35] Fischer S C L, Arzt E, Hensel R. Composite Pillars with a tunable interface for adhesion to rough substrates. ACS Appl Mater Interfaces 9(1): 1036-1044 (2017)

[36] Murphy M P, Sitti M. Waalbot: an agile small-scale wallclimbing robot utilizing dry elastomer adhesives. IEEE/ ASME Trans Mechatron 12(3): 330-338 (2007)

[37] Kim S, Spenko M, Trujillo S, Heyneman B, Santos D, Cutkosky M R. Smooth vertical surface climbing with directional adhesion. IEEE Trans Robotics 24(1): 65-74 (2008)

[38] Asbeck A, Dastoor S, Parness A, Fullerton L, Esparza N, Soto D, Heyneman B, Cutkosky M. Climbing rough vertical surfaces with hierarchical directional adhesion. In Proceedings of the 2009 IEEE International Conference on Robotics and Automation, Kobe, Japan, 2009: 2675-2680.

[39] Seo T, Sitti M. Tank-like module-based climbing robot using passive compliant joints. IEEE/ASME Trans Mechatron 18(1): 397-408 (2013)

[40] Henrey M, Ahmed A, Boscariol P, Shannon L, Menon C. Abigaille-III: A versatile, bioinspired hexapod for scaling smooth vertical surfaces. J Bionic Eng 11(1): 1-17 (2014)

[41] Breckwoldt W A, Daltorio K A, Heepe L, Horchler A D, Gorb S N, Quinn R D. Walking inverted on ceilings with wheel-legs and micro-structured adhesives. In Proceedings of the 2015 IEEE/RSJ International Conference on Intelligent Robots and Systems (IROS), Hamburg, Germany, 2015: 3308-3313.

[42] Ko H, Yi H, Jeong H E. Wall and ceiling climbing quadruped robot with superior water repellency manufactured using 3D printing (UNIclimb). Int J Precis Eng Manuf Green Technol 4(3): 273-280 (2017)

[43] Zhou M, Tian Y, Sameoto D, Zhang X J, Meng Y G, Wen S Z. Controllable interfacial adhesion applied to transfer light and fragile objects by using gecko inspired mushroom-shaped pillar surface. ACS Appl Mater Interfaces 5(20): 1013710144 (2013)

[44] Bae W G, Kim D, Suh K Y. Instantly switchable adhesion of bridged fibrillar adhesive via gecko-inspired detachment mechanism and its application to a transportation system. Nanoscale 5(23): 11876-11884 (2013)

[45] Gillies A G, Kwak J, Fearing R S. Controllable particle adhesion with a magnetically actuated synthetic gecko adhesive. Adv Funct Mater 23(26): 3256-3261 (2013)

[46] Song S, Sitti M. Soft grippers using micro-fibrillar adhesives for transfer printing. Adv Mater 26(28): 4901-4906 (2014)

[47] Song S, Drotlef D M, Majidi C, Sitti M. Controllable load sharing for soft adhesive interfaces on three-dimensional surfaces. PNAS 114(22): E4344-E4353 (2017)

[48] Shahsavan H, Salili S M, Jákli A, Zhao B X. Thermally active liquid crystal network gripper mimicking the self-peeling of gecko toe pads. Adv Mater 29(3): 1604021 (2017)

[49] Li S, Tian H M, Shao J Y, Liu H R, Wang D R, Zhang W T. Switchable adhesion for nonflat surfaces mimicking geckos' adhesive structures and toe muscles. ACS Appl Mater Interfaces 12(35): 39745-39755 (2020)

[50] Kizilkan E, Strueben J, Staubitz A, Gorb S N. Bioinspired photocontrollable microstructured transport device. Sci Robot 2(2): eaak9454 (2017)

[51] Li L, Liu Z Y, Zhou M, Li X X, Meng Y G, Tian Y. Flexible adhesion control by modulating backing stiffness based on jamming of granular materials. Smart Mater Struct 28(11): 115023 (2019)

[52] Xu Q, Wan Y Y, Hu T S, Liu T X, Tao D S, Niewiarowski P H, Tian Y, Liu Y, Dai L M, Yang Y Q, et al. Robust self-cleaning and micromanipulation capabilities of gecko spatulae and their bio-mimics. Nat Commun 6: 8949 (2015)

[53] Shui L Q, Jia L B, Li H B, Guo J J, Guo Z Y, Liu Y L, Liu Z, Chen $X$. Rapid and continuous regulating adhesion strength by mechanical micro-vibration. Nat Commun 11(1): 1583 (2020)

[54] Kim T, Park J, Sohn J, Cho D, Jeon S. Bioinspired, highly stretchable, and conductive dry adhesives based on 1D-2D hybrid carbon nanocomposites for all-in-one ECG electrodes. ACS Nano 10(4): 4770-4778 (2016)

[55] Drotlef D M, Amjadi M, Yunusa M, Sitti M. Bioinspired composite microfibers for skin adhesion and signal amplification of wearable sensors. Adv Mater 29(28): 1701353 (2017) 
[56] Autumn K. Gecko adhesion: Structure, function, and applications. MRS Bull 32(6): 473-478 (2007)

[57] Tian Y, Wan J, Pesika N, Zhou M. Bridging nanocontacts to macroscale gecko adhesion by sliding soft lamellar skin supported setal array. Sci Rep 3: 1382 (2013)

[58] Liu Z Y, Lu H Y, Zheng Y L, Tao D S, Meng Y G, Tian Y. Transient adhesion in a non-fully detached contact. Sci Rep 8: 6147 (2018)

[59] Cho H, Wu G X, Jolly J C, Fortoul N, He Z P, Gao Y C, Jagota A, Yang S. Intrinsically reversible superglues via shape adaptation inspired by snail epiphragm. PNAS 116(28): 13774-13779 (2019)

[60] Hawkes E W, Eason E V, Christensen D L, Cutkosky M R. Human climbing with efficiently scaled gecko-inspired dry adhesives. J R Soc Interface 12(102): 20140675 (2015)

[61] Bartlett M D, Croll A B, King D R, Paret B M, Irschick D J, Crosby A J. Looking beyond fibrillar features to scale gecko-like adhesion. Adv Mater 24(8): 1078-1083 (2012)

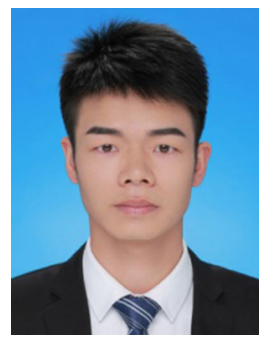

Xiaosong LI. He received his B.S. degree in mechanical engineering from Beijing Insititute of Technology, China. Now he is a Ph.D. candidate

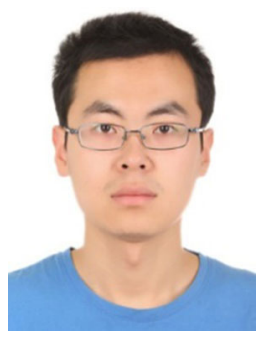

Pengpeng BAI. He received his Ph.D. degree at China University of Petroleum (Beijing), in 2017, majoring in materials science and engineering. Following a postdoctoral period at the State Key Laboratory of Tribology in Tsinghua University,

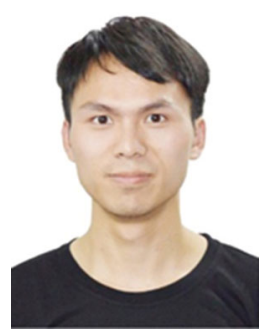

Xinxin LI. He received his B.S. degree in mechanical engineering from Huazhong University of Science and Technology, China. Now he is
[62] King D R, Bartlett M D, Gilman C A, Irschick D J, Crosby A J. Creating gecko-like adhesives for "real world" surfaces. Adv Mater 26(25): 4345-4351 (2014)

[63] Gilman C A, Imburgia M J, Bartlett M D, King D R, Crosby A J, Irschick D J. Geckos as springs: Mechanics explain across-species scaling of adhesion. PLOS One 10(9): e0134604 (2015)

[64] Hu H, Tian H M, Shao J Y, Wang Y, Li X M, Tian Y, Ding Y C, Lu B H. Friction contribution to bioinspired mushroomshaped dry adhesives. Adv Mater Interfaces 4(9): 1700016 (2017)

[65] Li X S, Pesika N, Li L, Li X X, Li Y Z, Bai P P, Meng Y G, Tian Y. Role of structural stiffness on the loading capacity of fibrillar adhesive composite. Extreme Mech Lett 41: 101001 (2020)

[66] Kim S, Sitti M, Hui C Y, Long R, Jagota A. Effect of backing layer thickness on adhesion of single-level elastomer fiber arrays. Appl Phys Lett 91(16): 161905 (2007)

in the Department of Mechanical Engineering, Tsinghua University, China. His current research interest is biomimetic adhesion and dexterous grasping technology.

he is now working as an associate researcher in the Department of Mechanical Engineering, Tsinghua University. He has published over 40 papers. His research interests include the interface science and technology, high temperature liquid lubricant, corrosion protection mechanism of metals, etc.

a Ph.D. candidate in the Department of Mechanical Engineering, Tsinghua University, China. His current research focuses on friction and bionic mechanisms. 


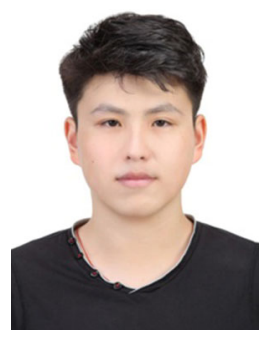

Lvzhou LI. He received his B.S. degree in electrical engineering and its automation from Jiangsu University, China. Now he is a

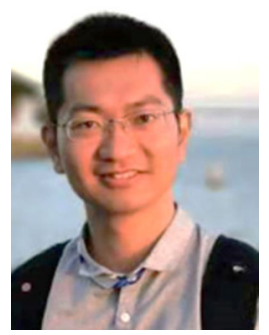

Yuanzhe LI. He received his B.S. degree in mechanical engineering from Xi'an Jiaotong University. Now he is a Ph.D. candidate in

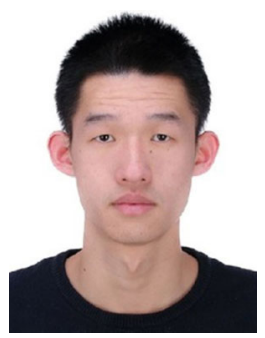

Hongyu LU. He received his B.S. degree from Beihang University in 2015, and received his Ph.D. degree from Tsinghua University in 2020.

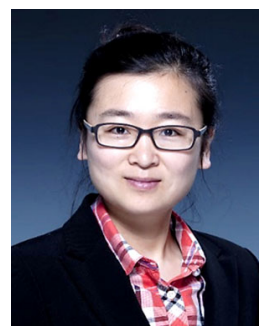

Liran MA. She received her B.S. degree from Tsinghua University in 2005, and received her Ph.D. degree from Tsinghua University in 2010. Following a postdoctoral period at the Weizmann Institute of Science in Israel, she is now working as an associate professor in the State Key Laboratory

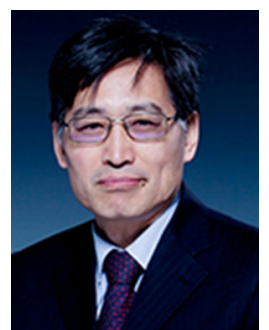

Yonggang MENG. He received his M.S. and Ph.D. degrees in mechanical engineering from Kumamoto University, Japan, in 1986 and 1989, respectively. He joined the State Key Laboratory of
Ph.D. candidate in the Department of Mechanical Engineering, Tsinghua University, China. His current research interest is adhesive soft robotics and variable stiffness systems.

the Department of Mechanical Engineering, Tsinghua University, China. His current research focuses on intermolecular force and tactile friction.

His research interest includes microforce measurement and biomimetic. Now he is working in Huawei Technologies Co., Ltd.

of Tribology, Tsinghua University. Her interests in tribology have ranged from aqueous lubrication and hydration lubrication to the liquid/solid interface properties. She has published over 50 papers. Her honors include the Hinwin Doctoral Dissertation Award (2011), the Maple Leaf Award for Outstanding Young Tribologists (2015), and Chang Jiang Scholars Program-Young Professor Award (2015).

Tribology at Tsinghua University in 1990. His current position is a professor, and his research areas cover the tribology of MEMS and hard disk drives, active control of friction and interfacial phenomena, and nanomanufacturing. 


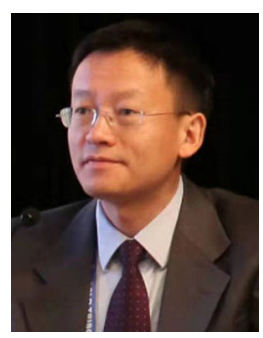

Yu TIAN. He is a professor and director of the State Key Laboratory of Tribology at Tsinghua University of China. He gained his B.S. and Ph.D. degrees in mechanical engineering at Tsinghua University in 1998 and 2002, respectively. Subsequently, he joined the State Key Laboratory of Tribology. He was a postdoc at the University of California, Santa Barbara with professor Jacob ISRAELACHVILI from 2005 to 2007. His research interest is the science and technology at the interface of physics, materials, engineering, and biology to understand the physical laws of adhesion, friction, and rheology to implement technological inventions to benefit the society. He has published over 150 peer-reviewed journal papers. He has received the Youth Science and Technology Award of China (2016), the Yangtze River Scholars Distinguished Professor (2015-2019), the National Natural Science Foundation for Distinguished Young Scientists of China (2014), the Wen Shizhu-Maple Award-Young Scholar Award (2012), the Young Scholar Achievement Award of the Society of Mechanical Engineering of China (2011), Outstanding Young Scholar Award of the Chinese Tribology Institute (2009), and the National Excellent Doctoral Dissertation of China (2004). 